\title{
Upregulation of Dopamine D2 Receptors in the Nucleus Accumbens Indirect Pathway Increases Locomotion but Does Not Reduce Alcohol Consumption
}

\author{
Eduardo F Gallo ${ }^{1,2}$, Michael C Salling ${ }^{3}$, Bo Feng ${ }^{1,2}$, Jose A Morón ${ }^{3}$, Neil L Harrison ${ }^{1,3}$, Jonathan A Javitch,1,2 \\ and Christoph Kellendonk ${ }^{*, 1,2}$
}

'Departments of Psychiatry and Pharmacology, College of Physicians and Surgeons, Columbia University, New York, NY, USA; ${ }^{2}$ Division of Molecular Therapeutics, New York State Psychiatric Institute, New York, NY, USA; ${ }^{3}$ Departments of Anesthesiology, College of Physicians and Surgeons, Columbia University, New York, NY, USA

\begin{abstract}
Brain imaging studies performed in humans have associated low striatal dopamine release and D2R binding with alcohol dependence. Conversely, high striatal D2R binding has been observed in unaffected members of alcoholic families suggesting that high D2R function may protect against alcohol dependence. A possible protective role of increased D2R levels in the striatum is further supported by preclinical studies in non-human primates and rodents. Here, we determined whether there is a causal relationship between D2R levels and alcohol intake. To this end, we upregulated D2R expression levels in the nucleus accumbens of the adult mouse, but selectively restricted the upregulation to the indirect striatal output pathway, which endogenously expresses D2Rs. After overexpression was established, mice were tested in two models of free-choice alcohol drinking: the continuous and intermittent access two-bottle choice models. As anticipated, we found that D2R upregulation leads to hyperactivity in the open field. Contrary to our expectation, D2R upregulation did not reduce alcohol intake during continuous or intermittent access or when alcohol drinking was tested in the context of aversive outcomes. These data argue against a protective role of accumbal indirect pathway D2Rs in alcohol consumption but emphasize their importance in promoting locomotor activity.

Neuropsychopharmacology (2015) 40, 1609-1618; doi:10.1038/npp.2015.II; published online 4 February 20I5
\end{abstract}

\section{INTRODUCTION}

There is considerable evidence implicating dopamine D2 receptor (D2R) function in the striatum in substance use disorders. Brain imaging studies performed in humans using positron emission tomography have consistently shown decreases in striatal dopamine release and D2R binding in alcohol dependence (Hietala et al, 1994; Volkow et al, 1996; Heinz et al, 2004; Martinez et al, 2005). The presumed decrease in D2R signaling that would be expected from reduced receptor levels may have important clinical implications. In alcoholics, low D2R binding is predictive of increased craving for alcohol, which correlates with a greater propensity to relapse (Heinz et al, 2005). In contrast, the high striatal D2R binding found in unaffected members of alcoholic families suggests that high D2R function may be protective against alcohol dependence (Volkow et al, 2006).

*Correspondence: Dr C Kellendonk or Dr JA Javitch, Columbia University/New York State Psychiatric Institute, I05 I Riverside Drive, Unit 19, New York, NY 10032, USA, Tel: + I 6467748602 or + | 646774 8600, Fax: + 6467748438 ,

E-mail: ck49I@columbia.edu or jaj2@columbia.edu

Received 2I August 2014; revised II November 2014; accepted 23

November 2014; accepted article preview online 12 January 2015
On the basis of human studies, it has been difficult to determine whether decreased signaling at the striatal D2R reflects vulnerability to developing an addiction that was present before drug use, or whether it is a consequence of chronic drug intake. Support for both of these mechanisms can be found in animal models. Lower D2R availability measured by positron emission tomography in drug-naïve non-human primates predicts higher cocaine self-administration, but chronic self-administration also further lowers D2R availability (Morgan et al, 2002; Nader et al, 2006). Lower D2R availability in the ventral striatum is also associated with increased cocaine self-administration in rats that show impulsivity as a behavioral trait (Dalley et al, 2007). In the context of alcohol addiction, striatal D2R density is reduced in rat and mouse strains genetically selected for high alcohol consumption and the reductions in D2R density are observed before any exposure to alcohol (Stefanini et al, 1992; McBride et al, 1993; Bice et al, 2008).

These studies suggest that striatal D2R levels in drugnaïve animals can predict drug use at a later time point. However, establishing causality is critical as it may inform new strategies for preventing or reversing excessive drug use. Thanos et al $(2001,2004,2005)$ have investigated this question by transiently upregulating D2R expression levels in the nucleus accumbens (NAc) in rodents using 
adenoviral-mediated gene transfer. They found that D2R upregulation in the NAc core transiently reduced established alcohol drinking and preference in both wild-type and alcohol-preferring rodents. Although these results have significant implications for understanding the role of D2Rs in alcoholism, some important issues remain to be addressed. First, in these studies, D2Rs were overexpressed after alcohol drinking was already established, thereby demonstrating that D2R overexpression has direct consequences on ongoing excessive alcohol intake. However, whether increasing D2R in drug-naïve animals also confers later protection against excessive alcohol consumption is currently unknown and needs to be addressed. Second, the viral vector used in these previous studies is only transiently expressed, making it impossible to determine whether the acute effect of D2R overexpression on alcohol consumption would be maintained. Third, the neuronal cell type in which $\mathrm{D} 2 \mathrm{R}$ overexpression may confer protection is unclear because the viral vector that was used can infect any cell type including medium spiny projection neurons (MSNs) as well as interneurons.

Striatal MSNs are organized into the direct and indirect projection pathways. The direct pathway predominantly expresses D1Rs and projects monosynaptically to the basal ganglia output nuclei, the internal segment of the globus pallidus and the substantia nigra pars reticulata. In contrast, the indirect pathway predominantly expresses D2Rs and modulates globus pallidus/substantia nigra pars reticulata output through a polysynaptic circuit via the external segment of the GP (Gerfen and Surmeier, 2011). Both pathways are functionally opposing with regard to thalamocortical activation and are therefore often referred to as 'Go' and 'NoGo' pathway. Although this separation is less distinct in the NAc compared with the dorsal striatum (Bertran-Gonzalez et al, 2008), activity in the direct and indirect pathway of the NAc have opposing influences on behaviors. In the context of drug addiction, sensitization and conditioned place preference to psychostimulants are generally promoted by the direct pathway but inhibited by the indirect pathway (Durieux et al, 2009; Ferguson et al, 2010; Lobo et al, 2010; Bock et al, 2013).

Thus, based on the previous work by Thanos et al and the known circuitry of the striatal output pathways, we sought to address two key questions left unanswered by previous studies: (i) When induced prior to alcohol exposure, does overexpression of D2Rs confer protection against alcohol drinking? (ii) Is overexpression that is selectively localized to the neurons of the indirect pathway, which endogenously express D2Rs, sufficient to provide protection? To this end, we used an adeno-associated (AAV) viral vector in combination with the Cre/loxP recombination system to produce long-lasting, stable D2R overexpression in the indirect pathway and measured its effect on subsequent alcohol consumption in two models of free-choice alcohol drinking.

\section{MATERIALS AND METHODS}

\section{Mice}

All procedures were approved by the Institutional Animal Care and Use Committee at Columbia University. Adult male D2-Cre BAC transgenic mice (ER44 line; GENSAT) backcrossed $>10$ generations onto the C57BL/6J background were singly housed under 12-h light/dark cycles with ad libitum food access during behavioral studies.

\section{AAV Generation and Delivery}

The AAV2/1-hSyn-DIO-D2R(L)-IRES-mVenus virus was generated by sub-cloning the sequence of interest (Figure 1a) under the human synapsin I promoter into the pAAV-EF1a-DIO-hChR2 plasmid (Addgene) packaged by Vector BioLabs (Philadelphia, PA). The control AAV2/ 5-hSyn-DIO-EGFP was purchased from the UNC Vector Core (University of North Carolina, Chapel Hill, NC). Mice were bilaterally injected at 8-9 weeks of age with $0.44 \mu \mathrm{l}$ of D2R or EGFP AAVs using stereotactic coordinates: (AP, $+1.7 \mathrm{~mm}$; ML, $\pm 1.2 \mathrm{~mm}$; DV,$-3.9 \mathrm{~mm}$ ). All experiments were conducted 4 weeks post injection.

\section{Immunohistochemistry and Radioligand Binding Assay}

Mice were deeply anesthetized and transcardially perfused with ice-cold $4 \%$ paraformaldehyde in PBS (Sigma, St Louis, $\mathrm{MO})$. Brains were harvested and postfixed overnight. Freefloating $30-\mu \mathrm{m}$ coronal sections were obtained using a Leica VT2000 vibratome (Richmond, VA). After incubation in blocking solution ( $10 \%$ fetal bovine serum, $0.5 \%$ bovine serum albumin in $0.5 \%$ TBS-Triton $\mathrm{X}-100$ ) for $1 \mathrm{~h}$ at room temperature, sections were labeled overnight at $4{ }^{\circ} \mathrm{C}$ with primary antibodies against GFP/mVenus (1:1000; Abcam, Cambridge, MA); D2R (1:500; in-house); choline acetyltransferase ( $1: 100$; Millipore, Temecula, CA); Cre-recombinase (1:3000; in-house), DsRed ( $1: 250$; Clontech, Mountain View, CA). Sections were incubated with fluorescent secondary antibodies for $1 \mathrm{~h}$ at $\mathrm{RT}$ and mounted on slides and coverslipped with Vectashield (Vector, Burlingame, CA). Digital images were acquired using a Nikon Meta Inverted confocal microscope and processed with $\mathrm{NIH}$ Image $J$ and Adobe Photoshop software. A $\left[{ }^{3} \mathrm{H}\right] \mathrm{N}$-methylspiperone binding assay was used to assess D2R ligand binding capacity in NAc membrane preparations, as previously done (Trifilieff et al, 2013).

\section{Locomotor Activity}

D2-Cre mice injected with D2R- or EGFP-expressing AAVs were tested in open field boxes equipped with infrared photobeams to measure locomotor activity (Med Associates, St. Albans, VT). Data were acquired using Kinder Scientific Motor Monitor software (Poway, CA) and expressed as total distance traveled $(\mathrm{cm})$ over $90 \mathrm{~min}$.

\section{Voluntary Two-Bottle Drinking Procedures}

Four weeks after AAV injection and 3 days after habituation to drinking water from two $50-\mathrm{ml}$ polycarbonate tubes, single-housed mice ( $n=8-9$ per group) were given access to two bottles outfitted with ball point stainless steel tubes (Ancare, Bellmore, NY), containing either 3\% ethanol in tap water $(\mathrm{v} / \mathrm{v})$ or tap water for 2 days. Each day, the bottle position was reversed to prevent location bias. For continuous access (CA), alcohol concentration was 

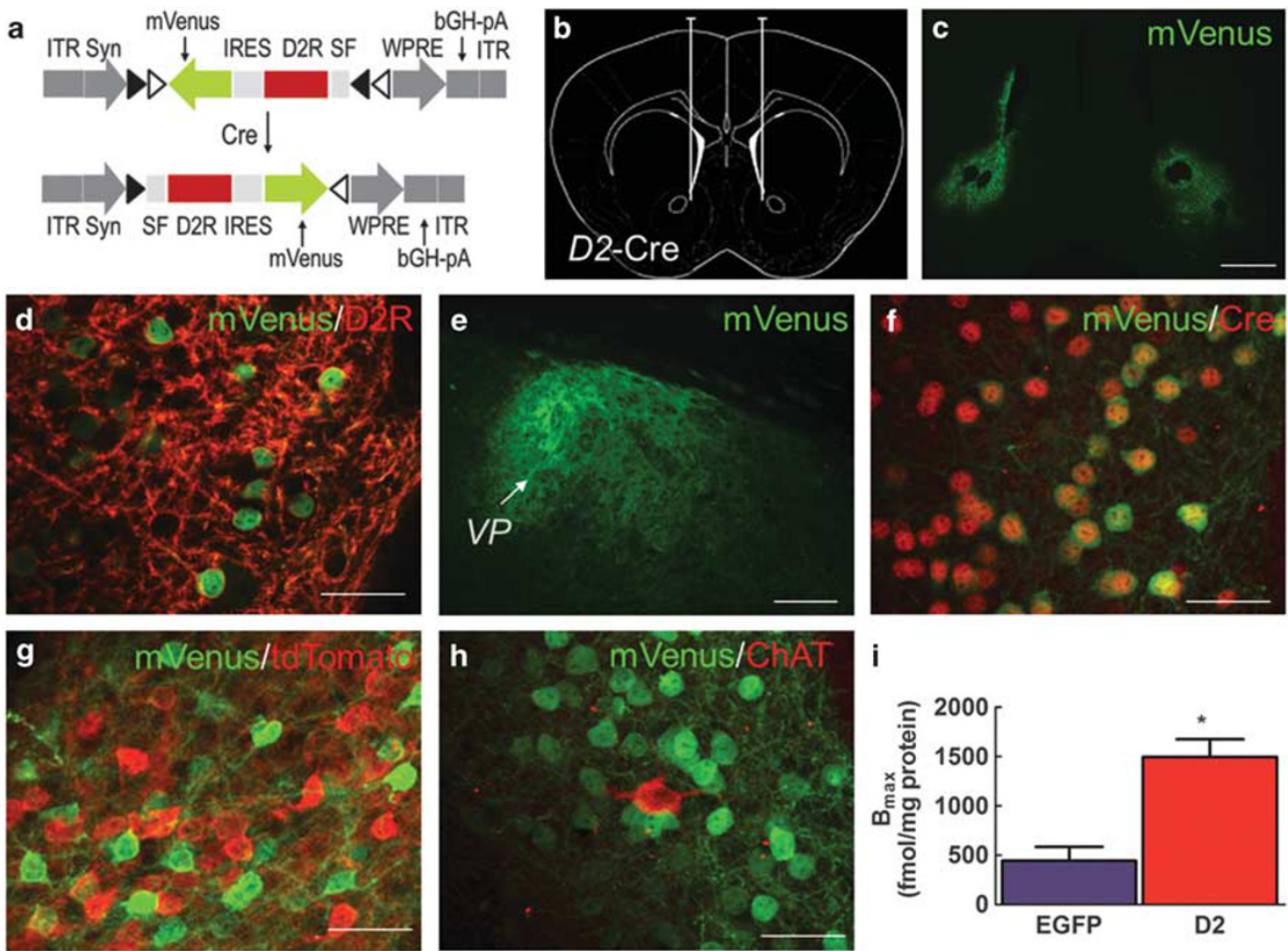

Figure I (a) AAV2/I vector encoding a double-floxed inverted open reading frame (DIO) expressing D2R and the YFP variant mVenus under control of the synapsin promoter. ITR, inverted terminal repeat; IRES, internal ribosome entry site; WPRE, woodchuck hepatitis virus posttranscriptional regulatory element; bGH-pA, bovine growth hormone poly-A tail. (b) Schematic representation depicting bilateral injection site of AAV into NAc of D2-Cre mice. (c) mVenus expression in NAc 4 weeks post injection is primarily restricted to the core region. Scale $=600 \mu \mathrm{m}$. (d) Co-localization of upregulated D2R (red) with $\mathrm{mVenus} \mathrm{(green)} \mathrm{was} \mathrm{observed} \mathrm{in} \mathrm{membrane-like} \mathrm{regions} \mathrm{and} \mathrm{processes.} \mathrm{Scale}=50 \mu \mathrm{m}$. (e) Higher magnification image showing mVenus-labeled fibers in the ventral pallidum (VP), projection target of NAc indirect pathway. Scale $=150 \mu \mathrm{m}$. (f) Double-labeling of Cre and mVenus shows that mVenus expression is restricted to Cre-positive cells in D2-Cre mice. Scale $=50 \mu \mathrm{m}$. (g) Rare co-localization of tdTomato (DI-MSNs) and Venus (D2-MSNs overexpressing D2Rs) in the NAc of D2-Cre $\times$ D I-tdTomato mice 4 weeks after the AAV-D2R injection. (h) Choline acetyltransferase (ChAT), a marker for cholinergic interneurons rarely colocalizes with GFP-positive cells, in agreement with previous work in D2-Cre mice (Kravitz et al, 2010$)$. Scale $=50 \mu \mathrm{m}$. (i) Ligand binding analysis with the D2 receptor antagonist $\left.{ }^{3} \mathrm{H}\right] \mathrm{N}$-methyl-spiperone in NAc membranes shows significant upregulation in D2R $\mathrm{NAclnd}$ mice.

increased every 2 days in $3 \%$ increments until $30 \%$ for a total of 3 weeks. Mice and bottles were weighed daily to determine ethanol consumption and preference values over $24 \mathrm{~h}$. Drip values were also obtained daily from bottles in an empty control cage and subtracted from the drinking values. Mice were also tested for their preference for either sucrose, saccharin or quinine hemisulfate (Sigma, St Louis, MO) $v s$ water in a CA 2-bottle choice procedure. Saccharin and quinine experiments were done 8 and 10 weeks after $\mathrm{AAV}$ injection and lasted for 4 and 8 days, respectively. Sucrose experiments were run 4 weeks post injection and lasted 16 days. For the intermittent access (IA) two-bottle procedure, a different cohort of mice ( $n=7-9$ per group) was given every-other-day access to both $15 \%$ ethanol (v/v) and water as described (Melendez, 2011), 8 weeks post injection. A total of 10 IA sessions were conducted over 6 weeks. Each session consisted of two non-consecutive days of alcohol exposure; each day with the bottle locations reversed. Two water bottles were presented on intervening days. Quinine was introduced in the $15 \%$ alcohol solution immediately after the 10 IA sessions and its concentration increased with each subsequent session over 4 weeks.

\section{Data Analysis}

Data are expressed as mean \pm SEM. Students' $t$-tests were used to compare between two groups. Multiple comparisons were evaluated by one-way or two-way repeated measures ANOVA, using GraphPad Prism software. Statistical significance was considered for $p<0.05$.

\section{RESULTS}

Viral D2R Upregulation is Selective for D2R-Containing MSNs of the Adult NAc

We generated a Cre recombinase (Cre)-dependent AAV, which encodes a double-inverted open reading frame (DIO) containing the Drd2 gene (long isoform), an internal ribosome entry site (IRES), and the YFP variant mVenus under the control of a synapsin promoter (Figure 1a). In order to selectively upregulate D2Rs in indirect pathway D2R-expressing MSNs (D2-MSNs), we bilaterally injected this vector in the NAc of adult D2-Cre mice (Figure $1 \mathrm{~b}$ ). After 4 weeks, immunohistochemical labeling of mVenus 
revealed Cre-dependent viral expression in the NAc, predominantly in the core sub-region (Figure 1c). Expression was stable for at least 6 month after injection (Supplementary Figure S1). D2Rs and mVenus were coexpressed within cells with D2R expression enriched in neuropil (Figure 1d). Dense labeling of axonal terminals was observed in the projection target region of NAc D2-MSNs, the ventral pallidum (Figure 1e). Selective targeting of viral expression to D2-MSNs was confirmed by Cre co-labeling (Figure 1f). A small percentage (4.7\%) of mVenus-positive cells co-expressed tdTomato in D2-Cre x D1-tdTomato mice (Figure 1g). This degree of co-expression is expected from published reports from our group and others which estimate that $\sim 3-6 \%$ of NAc core neurons co-express D1Rs and D2Rs (Bertran-Gonzalez et al, 2008; Frederick et al, 2015). Striatal cholinergic interneurons are known to express D2Rs, so we examined whether cells expressing the cholinergic marker choline acetyltransferase were transduced by our vector. In agreement with previous work using this D2-Cre line (Kravitz et al, 2010), we found very few choline acetyltransferase-positive cells within the virustransduced regions (Figure $1 \mathrm{~h}$ ). Only $5.6 \%$ of choline acetyltransferase-positive cells co-expressed mVenus or Cre (7.7\%). Further, we quantified D2R levels in NAc membranes using a $\left[{ }^{3} \mathrm{H}\right] \mathrm{N}$-methyl-spiperone binding assay (Figure 1i). Viral D2R upregulation in NAc led to $\sim$ threefold increase in binding capacity $\left(B_{\max }=1498.0\right.$ fmol/mg protein) compared with EGFP-expressing NAc $\left(B_{\max }=443.4 ; t=4.7, p=0.01, n=6\right.$ mice (3 samples) per group).

\section{D2R Upregulation in NAc D2-MSNs Leads to Hyperlocomotion}

To determine whether indirect pathway-selective D2R

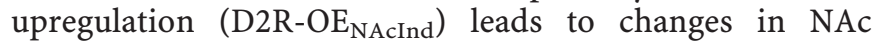
function in vivo, we first examined whether our manipulation would alter the balance of striatal control over locomotor activity. In the open field, D2R-OE $\mathrm{NAcInd}_{\text {mice }}$ exhibited a near twofold increase $(t=8.4, p<0.0001 ; n$ $=23-24$ per group) in total distance traveled across the 90 min examined compared with control D2-Cre mice injected with a Cre-dependent AAV expressing EGFP $\left(\right.$ EGFP $_{\text {NAcInd; }}$ Figure $2 \mathrm{a}$ and $\left.\mathrm{b}\right)$. The D2R-OE $\mathrm{EAcInd}_{\text {mice }}$ also displayed increased distance traveled in the center of the box and rearing behavior (Figure 2c-e; $t=3.0, p<0.005$ and $t=2.6, p<0.05$, respectively). These results show that increased D2R levels in the NAc indirect pathway lead to hyperlocomotion.

D2R Overexpression Increases Consumption of Fluids,
Including Alcohol, in a CA Model of Voluntary Drinking

We tested the effect of D2R overexpression in the NAc on alcohol-related behavior using the well-documented twobottle choice model of voluntary alcohol drinking (Richter and Campbell, 1940), in which mice drink freely from two bottles (alcohol $v s$ water). Specifically, we examined ethanol intake and preference following CA to a range of increasing ethanol concentrations (3-30\%) over the course of 3 weeks. Although intoxicating blood ethanol concentrations are rarely achieved with the CA model (Crabbe et al, 2011), this procedure has been valuable for characterizing basic drinking patterns across different mouse strains (Blednov et al, 2010).

With CA to increasing ethanol concentrations, both $\mathrm{EGFP}_{\mathrm{NAcInd}}$ and $\mathrm{D} 2 \mathrm{R}-\mathrm{OE}_{\mathrm{NAcInd}}$ mice progressively increased their daily alcohol intake, yet D2R-OE $\mathrm{NAcInd}_{\text {mice consumed }}$ significantly more alcohol (Figure $3 \mathrm{a}$ and Supplementary Figure $\mathrm{S} 2 ; \mathrm{F}_{(1,15)}=14.3, p<0.005$, two-way RM ANOVA, $n=8-9$ mice per group). Despite their increased alcohol intake, preference for alcohol over water was not altered in $\mathrm{D} 2 \mathrm{R}-\mathrm{OE}_{\mathrm{NAcIn}}$ mice relative to controls (Figure $3 \mathrm{~b}$ ). In agreement with previous work in the C57Bl6 strain (Blednov et al, 2010), both groups showed peak alcohol preference between 9 and 12\% ethanol and subsequently adjusted their preference at the higher ethanol doses. D2R-OE $\mathrm{NACInd}_{\text {mice }}$ also exhibited a significant increase in total fluid consumption across both bottles $\left(\mathrm{F}_{(1,15)}=31.8, p<0.0001\right)$ and a small decrease in body weight compared with controls $\left(\mathrm{F}_{(1,15)}=3.93, p=0.066\right.$; Figure $3 \mathrm{c}$ and $\left.\mathrm{d}\right)$. The increase in total fluid intake was evident even when two water bottles were presented prior to the introduction of ethanol (Figure 3c), demonstrating that the increased drinking behavior was not specific to alcohol.

To further examine this question, we tested drinking behavior in mice following pairings of sucrose, saccharin, or quinine vs water. Compared with controls, sucrose consumption was significantly higher in the D2R-OE $\mathrm{NACInd}$ group (Figure $3 e, \mathrm{~F}_{(1,15)}=12.05, p<0.005$ ). Saccharin and quinine intake were similarly increased, although not significantly (Figure $3 f$ and $g$; saccharin: $F_{(1,15)}=0.99$, $p=0.336$; quinine: $\left.\mathrm{F}\left({ }_{1,15}\right)=2.56, p=0.13\right)$. Preference tests for all three substances at increasing doses-as with alcohol-showed no significant differences between groups (Figure $3 \mathrm{~h}-\mathrm{j}$ ). Together, the drinking data from the CA procedure suggest that D2R overexpression in D2-MSNs leads to an increase in fluid consumption that is not specific to alcohol. In addition, D2R overexpression does not alter gustatory preferences for sweet or bitter tastes (Figure $3 \mathrm{~h}-\mathrm{j}$ ) known to strongly influence alcohol consumption (Blednov et $a l, 2008)$.

D2R Overexpression does not Modulate the Escalation of Alcohol Consumption in an IA Two-Bottle Choice Procedure

The CA two-bottle choice is limited in its ability to model important aspects of alcohol dependence, including escalation of drinking to intoxicating levels following periods of alcohol deprivation (Hwa et al, 2011). Therefore, we used an IA two-bottle procedure in which mice are given access to a fixed alcohol dose on alternating days over several weeks. The alternation between alcohol exposure and deprivation has been shown to lead to a rapid escalation in alcohol consumption in mice (Hwa et al, 2011; Melendez, 2011). In fact, the IA model is associated with withdrawal-like effects and leads to intoxicating blood alcohol levels, consistent with a dependent phenotype (Hwa et al, 2011).

We tested whether D2R upregulation in D2-MSNs would alter alcohol drinking of $15 \%$ alcohol when presented every other day for 6 weeks. Confirming the effectiveness of the IA procedure in leading to escalation of alcohol drinking, we found that both groups significantly increased their 
a
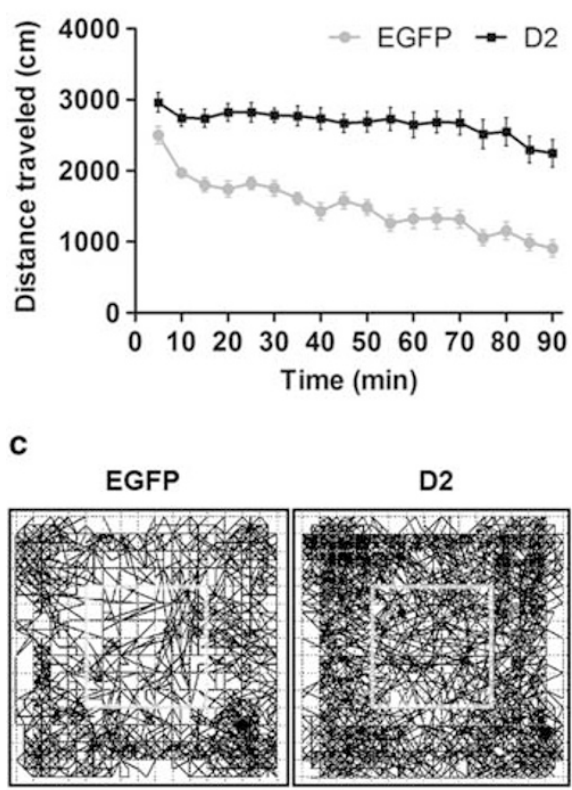

b
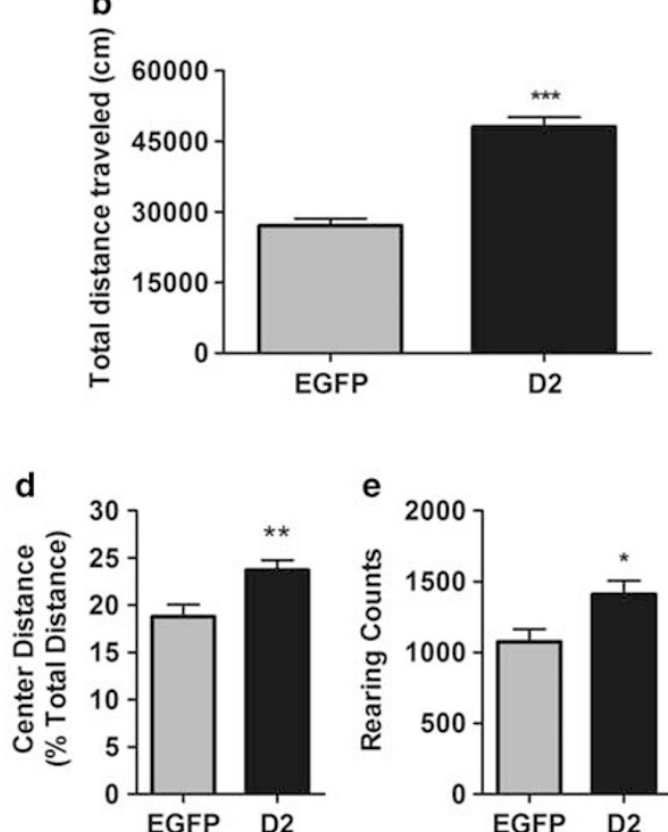

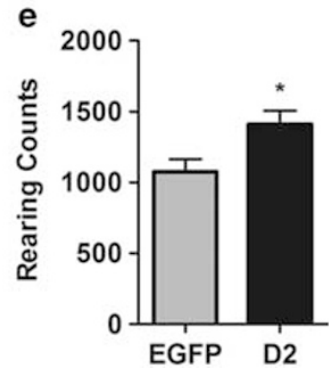

Figure 2 (a) Distance traveled in 5-min bins over a 90-min period by mice expressing EGFP or D2Rs in indirect pathway NAc MSNs. (b) Mean total distance traveled over the 90-min test period. (c) Representative horizontal activity tracks for the initial 45 min. Inner box represents the center zone. (d) Center distance, calculated as a percentage of the total distance, was significantly increased. (e) D2R-OE behavior. *Statistically significant.

alcohol intake throughout the 10 IA sessions, with control and $\mathrm{D} 2 \mathrm{R}-\mathrm{OE}_{\mathrm{NAcInd}}$ mice achieving mean doses of $17.3 \mathrm{mg} / \mathrm{kg}$ (51.9\% increase) and $18.9 \mathrm{mg} / \mathrm{kg}(41.5 \%$ increase), respectively (Figure 4a and b; EGFP: $t=11.4, p<0.0001$; D2: $t=3.55, p<0.01, n=7-9$ per group). However, we found no overall difference between groups. D2R-OE $\mathrm{NAcInd}_{\text {mice }}$ showed a small, but significant, reduction in preference for alcohol (Figure $4 \mathrm{c}, \mathrm{F}_{(1,14)}=6.3, p<0.05$, two-way $\mathrm{RM}$ ANOVA, $n=7-9$ per group). This effect on preference was due to increased water consumption because D2R-OE $\mathrm{NAcInd}$ exhibited increased total fluid consumption $\left(\mathrm{F}_{(1,14)}=20.8\right.$, $p<0.001$ ) but similar alcohol intake compared with controls (Figure 4d and Supplementary Figure S2). These data suggest that D2R upregulation in the NAc does not alter the escalation of alcohol consumption induced by this procedure.

\section{D2R Overexpression does not Modulate the De-Escalation of Alcohol Consumption when Drinking is Paired with a Negative Outcome}

Chronic alcohol intake in mice has been shown to lead to increased tolerance to the aversive effects of quinine when mixed with alcohol (Lesscher et al, 2010). Continued drinking of quinine-adulterated alcohol, despite its aversive properties, is thought to model the compulsive intake characteristic of alcohol dependence (Lesscher et al, 2010). We therefore determined whether excess D2Rs may affect how much alcohol the mice will continue to drink in the presence of increasing concentrations of quinine.

To this end, both groups of mice, which had been previously subjected to the IA procedure, were given access to $15 \%$ alcohol adulterated with concentrations of quinine ranging from 0.03 to $0.6 \mathrm{mM}$. Figure $5 \mathrm{a}$ and $\mathrm{b}$ show that drinking in both groups was largely resistant to quininecontaining alcohol at quinine concentrations that we and others had previously demonstrated to be aversive in the quinine preference test (Figure 3j; (Blednov et al, 2010), supporting the validity of the IA procedure in modeling compulsive-like aspects of alcohol dependence. A similar gradual reduction in alcohol consumption was observed in both groups with increasing quinine (Figure 5a). Preference for quinine-containing alcohol was reduced in the D2R$\mathrm{OE}_{\mathrm{NAcInd}}$ mice compared with controls (Figure 5B, $\left.\mathrm{F}_{(1,14)}=8.46, p<0.05\right)$. As in Figure $4 \mathrm{c}$, this genotype effect is likely due to the increased water intake, and not due to a reduction in alcohol intake (Figure $5 c$ and Supplementary Figure S2). While EtOH preference is reduced in D2R$\mathrm{OE}_{\mathrm{NAcInd}}$ mice, increasing quinine doses led to similar relative changes in preference in both groups (Supplementary Figure S3). Together, the intake and preference data suggest that D2R upregulation does not modulate de-escalation of drinking behavior in the face of an aversive outcome.

\section{DISCUSSION}

We used Cre-mediated somatic recombination with AAV gene transfer to selectively overexpress D2Rs in the indirect pathway MSNs of the NAc. We found that this manipulation led to a twofold increase in locomotor activity in the open field, but contrary to our original hypothesis, it did not lower alcohol intake in two different models of voluntary alcohol drinking. 

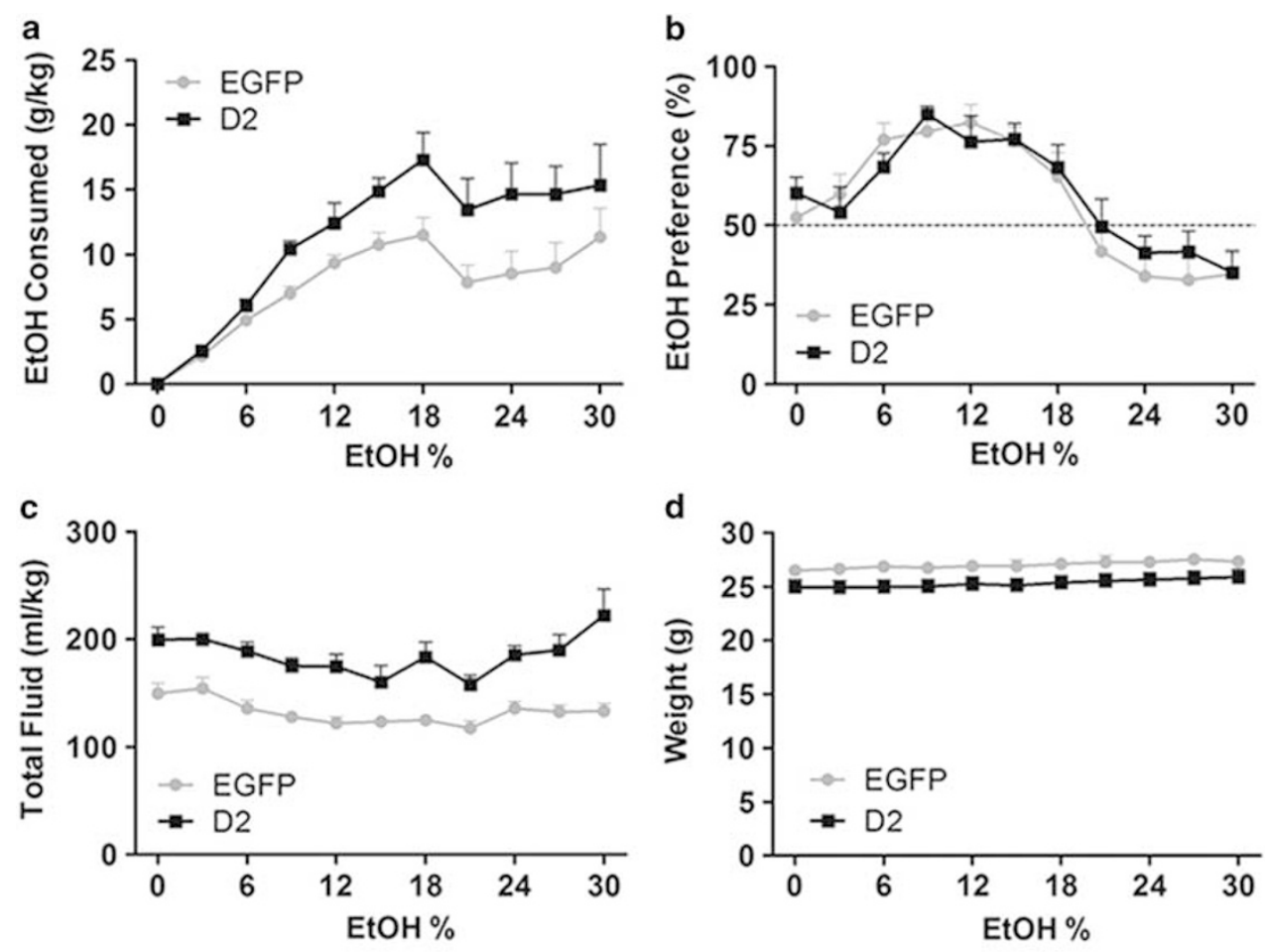

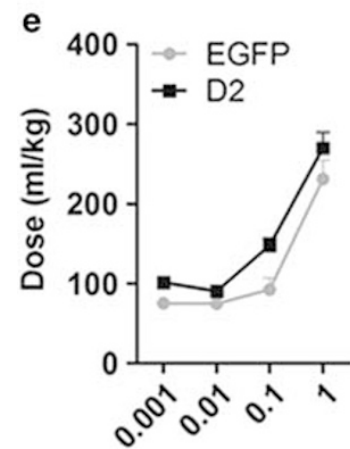

Sucrose $(\%)$

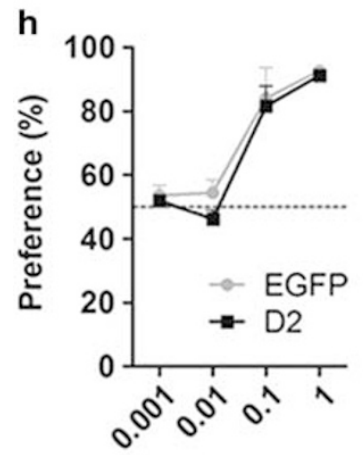

Sucrose (\%)
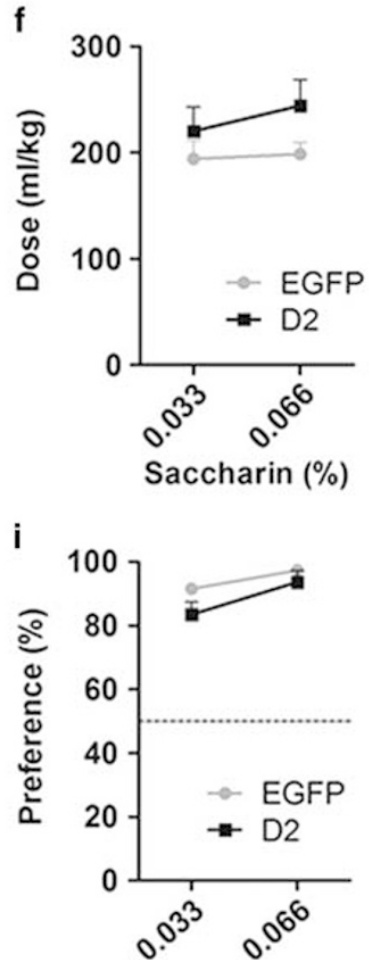

Saccharin $(\%)$ g
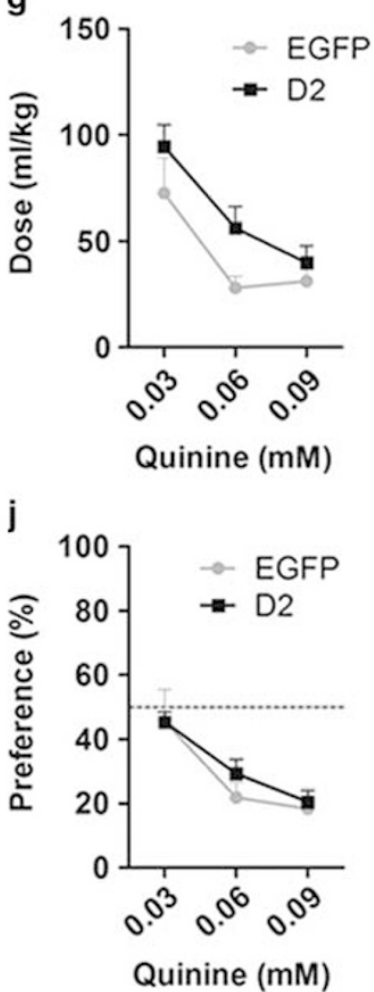

Figure 3 (a) Ethanol consumption is increased as a function of ethanol concentration ( $v / v$ ) in a continuous access, two-bottle choice procedure in both groups. Ethanol consumption is significantly higher in D2R-OENAclnd mice compared with controls expressing EGFP. Data for each ethanol concentration were obtained as an average of 2 days of drinking $(p<0.005)$. (b) Both groups similarly adjust their preference of ethanol over water across the different ethanol concentrations. (c) Total fluid consumption (ethanol + water), while stable in both groups, is increased in D2R-OENAclnd mice $(p<0.0000 \mathrm{l})$. (d) D2R overexpression results in lower body weights $(p=0.066)$. (e-g) Dose-dependent consumption of sucrose, saccharin, and quinine alone when tested against water. Sucrose consumption is significantly increased in D2R-OE $E_{N A c l n d}$ mice $(p<0.005)$. (h-j) Preference for sucrose, saccharin, or quinine over water is not altered by D2R upregulation. 

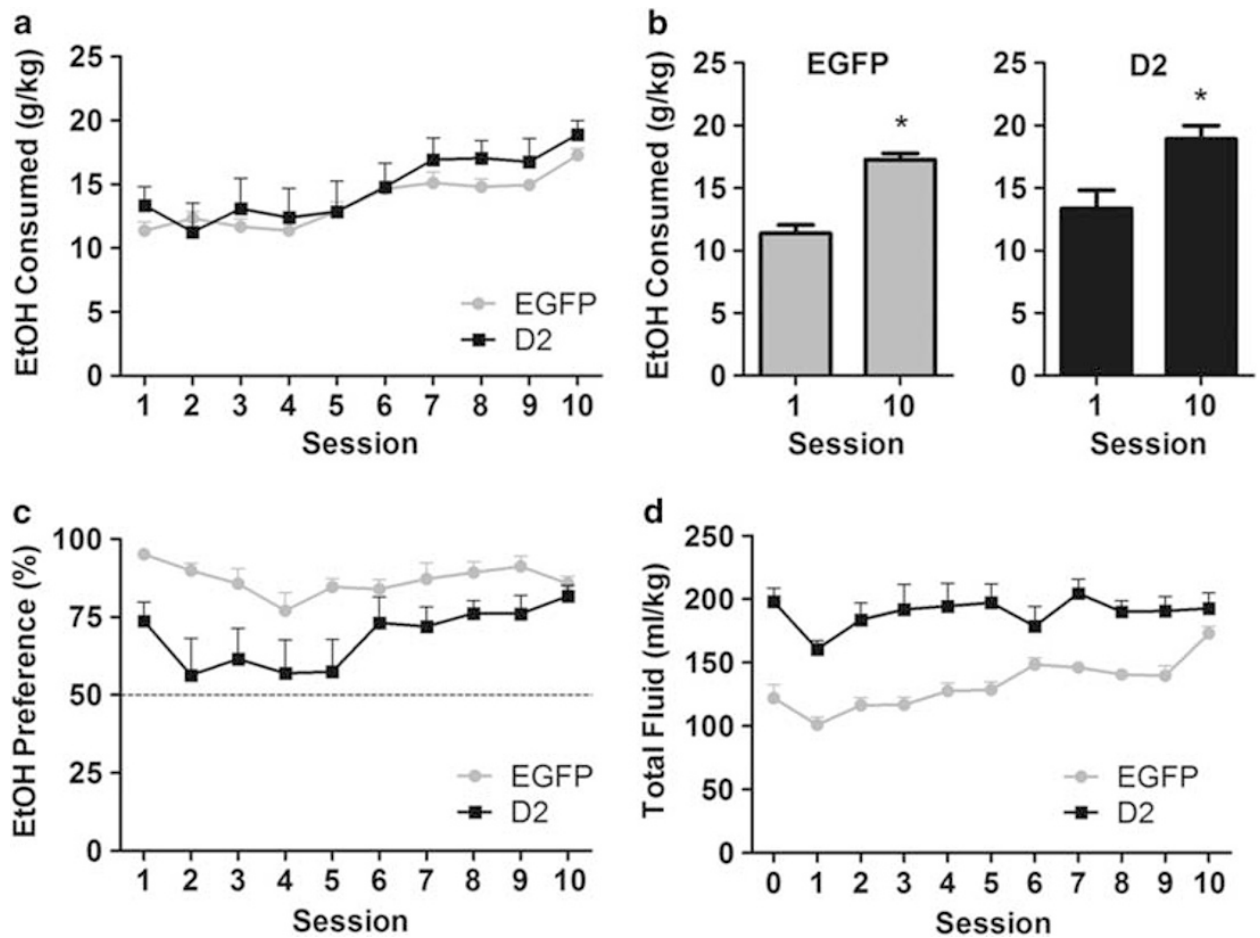

Figure 4 (a) Intermittent access (IA) to I5\% ethanol vs water leads to a progressive escalation of ethanol intake over 10 sessions (20 intermittent days of ethanol exposure). Each session reflects the average consumption over 2 days of alcohol drinking in which alcohol bottle position was alternated to prevent side bias. D2R upregulation does not alter escalation of ethanol consumption. (b) The IA procedure effectively leads to a significant increase in ethanol consumption in both groups between the first and last session. (c) Although both groups prefer 15\% ethanol over water, a reduction in preference was observed in the D2R-OE $E_{\text {Aaclnd }}$ group compared with controls $(p<0.05)$. (d) D2R upregulation also led to increased total fluid consumption $(p<0.00 \mathrm{I})$.

\section{D2R Upregulation in the Indirect Pathway Leads to Increased Locomotor Activity in the Open Field}

The direct and indirect striatal output pathways are thought to create a dynamic balance, exerting opposing but concerted actions on the control of movement, cognition and motivational processes (Gerfen and Surmeier, 2011; Cui et al, 2013). In line with this model, artificial stimulation of the indirect pathway using optogenetic tools inhibits locomotor activity in the open field (Kravitz et al, 2010; Cazorla et al, 2014). Conversely, selective lesion of the indirect pathway increases locomotion (Durieux et al, 2011). In the classical model of Parkinson's disease, D2Rs are thought to inhibit the indirect pathway activity and thus, D2R hyperfunction should disinhibit locomotor initiation and function (Gerfen and Surmeier, 2011). Our observations in the open field are consistent with this model. When D2Rs are upregulated in the indirect pathway, locomotor activity is enhanced. Our results differ from the described optogenetic and selective lesion studies in that we targeted the NAc core instead of the dorsomedial striatum (Kravitz et al, 2010; Durieux et al, 2011; Cazorla et al, 2014). However, numerous pharmacological studies using local infusions have demonstrated a role for dopamine in the NAc core in regulating open field activity (Pulvirenti et al, 1994; Swanson et al, 1997; Canales and Iversen, 2000; Baldo et al, 2002). Our results are consistent with these findings and suggest that the D2R in the accumbal indirect pathway is a critical regulator of locomotor activity and indicate that the virally expressed D2Rs are functional.

\section{D2R Upregulation in the Indirect Pathway does not Decrease Alcohol Drinking}

On the basis of the human imaging studies (Hietala et al, 1994; Volkow et al, 1996; Heinz et al, 2004; Martinez et al, 2005; Volkow et al, 2006), the psychostimulant studies in monkeys and rats (Morgan et al, 2002; Nader et al, 2006; Dalley et al, 2007), and the viral overexpression studies in rats and mice using adenoviruses (Thanos et al, 2001, 2004, 2005), we hypothesized that alcohol intake would be

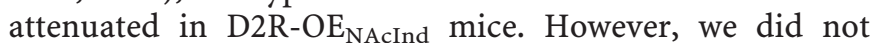
observe a decrease in alcohol consumption during either CA or IA procedures. We found a generalized increase in fluid consumption in $\mathrm{D} 2 \mathrm{R}-\mathrm{OE}_{\mathrm{NAcInd}}$ mice, which may be related to their higher activity as measured by hyperlocomotion. D2R upregulation resulted in increased alcohol consumption in the CA schedule, but did not alter intake in the IA schedule. The main difference between both models is that in the IA model, chronic cycling between periods of alcohol drinking and deprivation leads to enhanced escalation of consumption and thereby intoxicating blood ethanol concentrations. This is rarely observed using the CA model (Crabbe et al, 2011; Hwa et al, 2011). We suspect that this key aspect of the IA model may account for the lessened influence of the hyperingestive behavior over alcohol consumption in the IA model. Neither model, however, revealed a reduction in alcohol intake, arguing against a protective role of $\mathrm{D} 2 \mathrm{R}$ overexpression on escalation of voluntary alcohol drinking. Although preference for ethanol in the IA procedure was significantly reduced in $D 2 R_{N A c I n d}$ 

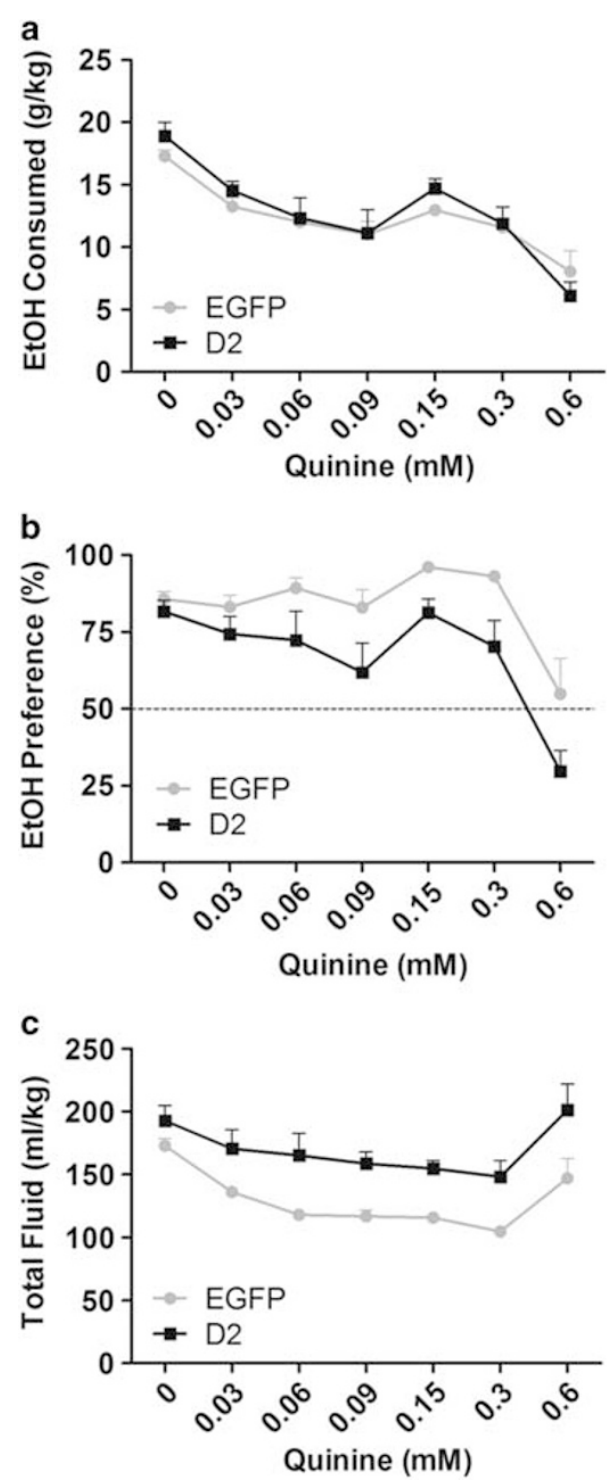

Figure 5 (a) After IO IA sessions of I5\% ethanol, quinine was faded into the ethanol solution beginning with concentrations that were aversive in alcohol-naive mice $(0.03-0.06 \mathrm{mM})$ up to $0.6 \mathrm{mM}$. Both groups showed a slow, but comparable decrease in their alcohol consumption with increasing quinine concentrations. (b) Although D2R-OE $\mathrm{NAclnd}_{\text {maintained }}$ a lower preference for alcohol $p<0.05$ both groups were similarly resistant to reducing their preference with increasing quinine concentrations, eventually decreasing preference at the highest quinine dose. (c) Total fluid consumption was stable across quinine doses, but remained higher in the D2R-OE $E_{\text {NAclnd }}$ group $(p<0.05)\left(F_{(1,14)}=8.13,\right)$.

mice, this decrease was driven by enhanced water intake, and not a reduction in alcohol intake. In line with this interpretation, in session 10 (Figure 4) where control and D2R-OE mice consume similar doses of total fluid (Figure 4d) and water (Supplementary Figure S2d), D2R$\mathrm{OE}_{\text {NAcInd }}$ mice did not show decreased preference for alcohol.

Furthermore, both the $\mathrm{D} 2 \mathrm{R}-\mathrm{OE}_{\mathrm{NAcInd}}$ and $\mathrm{EGFP}_{\mathrm{NAcInd}}$ mice were similarly sensitive to modulation of alcohol consumption and preference when the alcohol solution was adulterated with quinine. Consumption and preference

shifted to the water bottle only at the highest dose of quinine $(0.6 \mathrm{mM})$. This concentration was 10 -fold higher than the concentrations needed to curtail their drinking behavior in the absence of alcohol (Figure $3 g$ and $\mathrm{j}$ ), in line with previous reports in C57BL6 mice with a history of chronic alcohol consumption (Lesscher et al, 2010). The comparable tolerance to a quinine-adulterated alcohol by both groups suggests that $\mathrm{D} 2 \mathrm{R}$ overexpression in the NAc indirect pathway does not alter the impact of a negative outcome on elevated alcohol intake.

Our data seem at odds with the outcome of the adenovirus studies by Thanos et al (2001, 2004, 2005). There are several key differences in the objectives and design between these preceding studies and our work that may explain the apparent contradictions. First, the adenovirus experiments tested whether overexpression of D2Rs attenuates drinking after drinking behavior had already been established. In contrast, we used AAV to induce D2R overexpression before testing its effects on escalation of alcohol intake, in an effort to determine whether increased D2R levels confer protection against future drinking. It is conceivable that targeting D2R levels is only beneficial once drinking behavior or even alcohol dependence is established. Second, in the adenovirus experiments, D2Rs were overexpressed non-selectively in different cell types of the NAc, including the direct pathway that normally has only very limited expression of D2Rs (Bertran-Gonzalez et al, 2008). In contrast, using a Cre-LoxP- based strategy, we were able to restrict D2R overexpression to indirect pathway MSNs that express D2Rs. Given the lack of a protective effect by elevating D2R in this cell population, which includes MSNs that co-express D1Rs and D2Rs (Gerfen and Surmeier, 2011), it is possible that a D2R-based strategy aimed at attenuating drinking requires ectopic upregulation of D2Rs in direct pathway MSNs. This would be consistent with the classical model of basal ganglia circuitry and with observations in which inhibition of direct pathway MSNs impaired amphetamine sensitization (Ferguson et al, 2010) and cocaine place preference (Hikida et al, 2010). Third, the present experiments were designed to examine the long-term consequences of overexpressing D2R on drinking behavior. The time window of $\mathrm{D} 2 \mathrm{R}$ overexpression in the adenovirus experiments was only several days, whereas AAV expression in our study is stable through at least 6 months after transduction (Supplementary Figure S1). We found that chronic D2R upregulation in adult alcohol-naive mice, however, does not prevent the escalation of alcohol drinking. This outcome suggests that ongoing, high D2R levels in the indirect pathway may not reduce vulnerability to this particular behavior. A lack of protective effect by cell type-specific $\mathrm{D} 2 \mathrm{R}$ upregulation in this context does not rule out the possibility that our strategy could reduce vulnerability in D2R-deficient animals such as the high-alcohol preferring mouse strain (Bice et al, 2008). Moreover, high D2R levels in the indirect pathway may be protective against other drugs such as cocaine. However, a preliminary analysis of cocainerelated behaviors does not support this idea (Supplementary Figure S4).

Pharmacological studies using D2R agonists/antagonists in rodents or studies using D2R knockout mice have uncovered an involvement of D2Rs in a wide variety of 
alcohol-related behaviors. In many cases, the findings support a protective role of increased D2R function (Levy et al, 1991; Ng and George, 1994; Hodge et al, 1997; Bulwa et al, 2011). However, other reports using D2R antagonists or D2R knockout mice, for instance, show a reduction in alcohol self-administration or preference (Rassnick et al, 1992; Samson et al, 1993; Hodge et al, 1997; Phillips et al, 1998; Thanos et al, 2005), or no difference at all (Linseman, 1990). These inconsistencies may stem from differences in the specific drinking models, dopamine agonists/antagonist doses, or routes of administration (NAc vs systemic), emphasizing the need for highly targeted genetic approaches for the study of D2R function.

Our viral approach has enabled us to test the specific consequences of D2R upregulation in indirect pathway MSNs of the NAc on locomotor activity and drinking behavior, thus overcoming some of the limitations outlined above. Although indirect pathway D2R upregulation did not prevent alcohol drinking behavior, a protective action may require overexpression of indirect pathway D2Rs in other striatal regions, such as the NAc shell or the dorsal striatum. Our findings also raise the possibility that the D2R alterations that are thought to influence alcohol dependence in humans may not be confined to the indirect pathway of the NAc. In addition to MSNs, D2Rs are also found in striatal cholinergic interneurons and dopamine neuron terminals, where they regulate key functions, including dopamine release (Bello et al, 2011; Cachope et al, 2012; Threlfell et al, 2012). Future studies may benefit from implementing approaches like the ones described here to systematically characterize whether increased D2R levels in other cell types reduce susceptibility to alcohol drinking.

\section{FUNDING AND DISCLOSURE}

This work was supported by NIH T32 fellowship (T32DA016224) to E.F.G, NIH/NIAAA fellowship (5F32AA022028) for M.C.S., NIH grants R01DA025036 and R01DA027460 to J.A.M., NIH grant R01AA019801 to N.L.H., NIH grants MH54137 and DA022413 to J.A.J, and NIH grant MH093672 and Columbia University start-up funds to C.K. The authors declare no conflict of interest.

\section{ACKNOWLEDGEMENTS}

We thank Dr Nicholas Grahame and Dr Diana Martinez for their insightful comments on the manuscript. We also thank Claire Sunha Choi and Jeremy Sherman for their valuable technical assistance in conducting the 2-bottle choice experiments.

\section{REFERENCES}

Baldo BA, Sadeghian K, Basso AM, Kelley AE (2002). Effects of selective dopamine D1 or D2 receptor blockade within nucleus accumbens subregions on ingestive behavior and associated motor activity. Behav Brain Res 137: 165-177.

Bello EP, Mateo Y, Gelman DM, Noain D, Shin JH, Low MJ et al (2011). Cocaine supersensitivity and enhanced motivation for reward in mice lacking dopamine D2 autoreceptors. Nat Neurosci 14: 1033-1038.
Bertran-Gonzalez J, Bosch C, Maroteaux M, Matamales M, Hervé D, Valjent E et al (2008). Opposing patterns of signaling activation in dopamine D1 and D2 receptor-expressing striatal neurons in response to cocaine and haloperidol. The Journal of Neuroscience 8: 5671-5685.

Bice PJ, Liang T, Zhang L, Strother WN, Carr LG (2008). Drd2 expression in the high alcohol-preferring and low alcoholpreferring mice. Mamm Genome 19: 69-76.

Blednov YA, Ozburn AR, Walker D, Ahmed S, Belknap JK, Harris RA (2010). Hybrid mice as genetic models of high alcohol consumption. Behav Genet 40: 93-110.

Blednov YA, Walker D, Martinez M, Levine M, Damak S, Margolskee RF (2008). Perception of sweet taste is important for voluntary alcohol consumption in mice. Genes Brain Behav 7: 1-13.

Bock R, Shin JH, Kaplan AR, Dobi A, Markey E, Kramer PF et al (2013). Strengthening the accumbal indirect pathway promotes resilience to compulsive cocaine use. Nat Neurosci 16: 632-638.

Bulwa ZB, Sharlin JA, Clark PJ, Bhattacharya TK, Kilby CN, Wang $Y$ et al (2011). Increased consumption of ethanol and sugar water in mice lacking the dopamine D2 long receptor. Alcohol 45: 631-639.

Cachope R, Mateo Y, Mathur BN, Irving J, Wang HL, Morales M et al (2012). Selective activation of cholinergic interneurons enhances accumbal phasic dopamine release: setting the tone for reward processing. Cell Rep 2: 33-41.

Canales JJ, Iversen SD (2000). Psychomotor-activating effects mediated by dopamine $\mathrm{D}(2)$ and $\mathrm{D}(3)$ receptors in the nucleus accumbens. Pharmacol Biochem Behav 67: 161-168.

Cazorla M, de Carvalho FD, Chohan MO, Shegda M, Chuhma N, Rayport S et al (2014). Dopamine D2 receptors regulate the anatomical and functional balance of basal ganglia circuitry. Neuron 81: 153-164.

Crabbe JC, Harris RA, Koob GF (2011). Preclinical studies of alcohol binge drinking. Ann NY Acad Sci 1216: 24-40.

Cui G, Jun SB, Jin X, Pham MD, Vogel SS, Lovinger DM et al (2013). Concurrent activation of striatal direct and indirect pathways during action initiation. Nature 494: 238-242.

Dalley JW, Fryer TD, Brichard L, Robinson ES, Theobald DE, Laane K et al (2007). Nucleus accumbens D2/3 receptors predict trait impulsivity and cocaine reinforcement. Science 315: 1267-1270.

Durieux PF, Bearzatto B, Guiducci S, Buch T, Waisman A, Zoli M et al (2009). D2R striatopallidal neurons inhibit both locomotor and drug reward processes. Nature Neurosci 12: 393-395.

Durieux PF, Schiffmann SN, de Kerchove d'Exaerde A (2011). Differential regulation of motor control and response to dopaminergic drugs by $\mathrm{D} 1 \mathrm{R}$ and $\mathrm{D} 2 \mathrm{R}$ neurons in distinct dorsal striatum subregions. EMBO J 31: 640-653.

Ferguson SM, Eskenazi D, Ishikawa M, Wanat MJ, Phillips PE, Dong $\mathrm{Y}$ et al (2010). Transient neuronal inhibition reveals opposing roles of indirect and direct pathways in sensitization. Nature Neurosci 14: 22-24.

Frederick AL, Yano H, Trifilieff P, Vishwasrao HD, Biezonski D, Mészáros J et al (2015). Evidence against dopamine D1/D2 receptor heteromers. Mol Psychiatry (e-pub ahead of print 6 January 2015; doi:10.1038/mp.2014.166).

Gerfen CR, Surmeier DJ (2011). Modulation of striatal projection systems by dopamine. Annu Rev Neurosci 34: 441-466.

Heinz A, Siessmeier T, Wrase J, Buchholz HG, Grunder G, Kumakura Y et al (2005). Correlation of alcohol craving with striatal dopamine synthesis capacity and D2/3 receptor availability: a combined [18F]DOPA and [18F]DMFP PET study in detoxified alcoholic patients. Am J Psychiatry 162: 1515-1520.

Heinz A, Siessmeier T, Wrase J, Hermann D, Klein S, Grusser SM et al (2004). Correlation between dopamine $\mathrm{D}(2)$ receptors in the ventral striatum and central processing of alcohol cues and craving. Am J Psychiatry 161: 1783-1789. 
Hietala J, West C, Syvalahti E, Nagren K, Lehikoinen P, Sonninen P et al (1994). Striatal D2 dopamine receptor binding characteristics in vivo in patients with alcohol dependence. Psychopharmacology 116: 285-290.

Hikida T, Kimura K, Wada N, Funabiki K, Nakanishi S (2010). Distinct roles of synaptic transmission in direct and indirect striatal pathways to reward and aversive behavior. Neuron 66: 896-907.

Hodge CW, Samson HH, Chappelle AM (1997). Alcohol selfadministration: further examination of the role of dopamine receptors in the nucleus accumbens. Alcohol Clin Exp Res 21: 1083-1091.

Hwa LS, Chu A, Levinson SA, Kayyali TM, DeBold JF, Miczek KA (2011). Persistent escalation of alcohol drinking in C57BL/6J mice with intermittent access to $20 \%$ ethanol. Alcohol Clin Exp Res 35: 1938-1947.

Kravitz AV, Freeze BS, Parker PR, Kay K, Thwin MT, Deisseroth K et al (2010). Regulation of parkinsonian motor behaviours by optogenetic control of basal ganglia circuitry. Nature 466: 622-626.

Lesscher HM, van Kerkhof LW, Vanderschuren LJ (2010). Inflexible and indifferent alcohol drinking in male mice. Alcohol Clin Exp Res 34: 1219-1225.

Levy AD, Murphy JM, McBride WJ, Lumeng L, Li TK (1991). Microinjection of sulpiride into the nucleus accumbens increases ethanol drinking in alcohol-preferring $(\mathrm{P})$ rats. Alcohol Alcohol Suppl 1: 417-420.

Linseman MA (1990). Effects of dopaminergic agents on alcohol consumption by rats in a limited access paradigm. Psychopharmacology 100: 195-200.

Lobo MK, Covington HE 3rd, Chaudhury D, Friedman AK, Sun H, Damez-Werno D et al (2010). Cell type-specific loss of BDNF signaling mimics optogenetic control of cocaine reward. Science 330: 385-390.

Martinez D, Gil R, Slifstein M, Hwang DR, Huang Y, Perez A et al (2005). Alcohol dependence is associated with blunted dopamine transmission in the ventral striatum. Biol Psychiatry 58: 779-786.

McBride WJ, Chernet E, Dyr W, Lumeng L, Li TK (1993). Densities of dopamine D2 receptors are reduced in CNS regions of alcohol-preferring $P$ rats. Alcohol 10: 387-390.

Melendez RI (2011). Intermittent (every-other-day) drinking induces rapid escalation of ethanol intake and preference in adolescent and adult C57BL/6J mice. Alcohol Clin Exp Res 35: 652-658.

Morgan D, Grant KA, Gage HD, Mach RH, Kaplan JR, Prioleau O et al (2002). Social dominance in monkeys: dopamine D2 receptors and cocaine self-administration. Nat Neurosci 5: 169-174.

Nader MA, Morgan D, Gage HD, Nader SH, Calhoun TL, Buchheimer $\mathrm{N}$ et al (2006). PET imaging of dopamine D2 receptors during chronic cocaine self-administration in monkeys. Nat Neurosci 9: 1050-1056.
Ng GY, George SR (1994). Dopamine receptor agonist reduces ethanol self-administration in the ethanol-preferring C57BL/6J inbred mouse. Eur J Pharmacol 269: 365-374.

Phillips TJ, Brown KJ, Burkhart-Kasch S, Wenger CD, Kelly MA, Rubinstein $M$ et al (1998). Alcohol preference and sensitivity are markedly reduced in mice lacking dopamine D2 receptors. Nat Neurosci 1: 610-615.

Pulvirenti L, Berrier R, Kreifeldt M, Koob GF (1994). Modulation of locomotor activity by NMDA receptors in the nucleus accumbens core and shell regions of the rat. Brain Res 664: 231-236.

Rassnick S, Pulvirenti L, Koob GF (1992). Oral ethanol selfadministration in rats is reduced by the administration of dopamine and glutamate receptor antagonists into the nucleus accumbens. Psychopharmacology 109: 92-98.

Richter CP, Campbell KH (1940). Alcohol taste thresholds and concentrations of solution preferred by rats. Science 91: 507-508.

Samson HH, Hodge CW, Tolliver GA, Haraguchi M (1993). Effect of dopamine agonists and antagonists on ethanol-reinforced behavior: the involvement of the nucleus accumbens. Brain Res Bull 30: 133-141.

Stefanini E, Frau M, Garau MG, Garau B, Fadda F, Gessa GL (1992). Alcohol-preferring rats have fewer dopamine D2 receptors in the limbic system. Alcohol Alcohol 27: 127-130.

Swanson CJ, Heath S, Stratford TR, Kelley AE (1997). Differential behavioral responses to dopaminergic stimulation of nucleus accumbens subregions in the rat. Pharmacol Biochem Behav 58: 933-945.

Thanos PK, Rivera SN, Weaver K, Grandy DK, Rubinstein M, Umegaki $\mathrm{H}$ et al (2005). Dopamine D2R DNA transfer in dopamine D2 receptor-deficient mice: effects on ethanol drinking. Life Sci 77: 130-139.

Thanos PK, Taintor NB, Rivera SN, Umegaki H, Ikari H, Roth G et al (2004). DRD2 gene transfer into the nucleus accumbens core of the alcohol preferring and nonpreferring rats attenuates alcohol drinking. Alcohol Clin Exp Res 28: 720-728.

Thanos PK, Volkow ND, Freimuth P, Umegaki H, Ikari H, Roth G et al (2001). Overexpression of dopamine D2 receptors reduces alcohol self-administration. J Neurochem 78: 1094-1103.

Threlfell S, Lalic T, Platt NJ, Jennings KA, Deisseroth K, Cragg SJ (2012). Striatal dopamine release is triggered by synchronized activity in cholinergic interneurons. Neuron 75: 58-64.

Trifilieff P, Feng B, Urizar E, Winiger V, Ward RD, Taylor KM et al (2013). Increasing dopamine D2 receptor expression in the adult nucleus accumbens enhances motivation. Mol Psychiatry 18: 1025-1033.

Volkow ND, Wang GJ, Begleiter H, Porjesz B, Fowler JS, Telang F et al (2006). High levels of dopamine D2 receptors in unaffected members of alcoholic families: possible protective factors. Arch Gen Psychiatry 63: 999-1008.

Volkow ND, Wang GJ, Fowler JS, Logan J, Hitzemann R, Ding YS et al (1996). Decreases in dopamine receptors but not in dopamine transporters in alcoholics. Alcohol Clin Exp Res 20: 1594-1598.

Supplementary Information accompanies the paper on the Neuropsychopharmacology website (http://www.nature.com/npp) 